\title{
BMJ Open Continuous enteral nutrition compared with a maximal gastric vacuity strategy at the time of extubation in the intensive care unit: protocol for a non-inferiority cluster randomised trial (the Ambroisie Project)
}

To cite: Landais $\mathrm{M}$ Nay M-A, Auchabie J, et al. Continuous enteral nutrition compared with a maximal gastric vacuity strategy at the time of extubation in the intensive care unit: protocol for a non-inferiority cluster randomised trial (the Ambroisie Project). BMJ Open 2021;11:e041799. doi:10.1136/ bmjopen-2020-041799

- Prepublication history for this paper is available online. To view these files, please visit the journal online ().

Received 18 June 2020 Revised 21 April 2021 Accepted 28 April 2021
Check for updates

(C) Author(s) (or their employer(s)) 2021. Re-use permitted under CC BY-NC. No commercial re-use. See rights and permissions. Published by BMJ.

For numbered affiliations see end of article.

Correspondence to Dr Mickael Landais; mlandais@ch-lemans.fr

\section{ABSTRACT}

Introduction Fasting is frequently imposed to patients before extubation in the intensive care unit based on scheduled surgery guidelines. This practice has never been evaluated among critically ill patients and may delay extubation, increase nursing workload and reduce caloric intake. We are hypothesising that continuous enteral nutrition until extubation represents a safe alternative compared with fasting prior to extubation in the intensive care unit.

Methods and analysis Adult patients ventilated more than 48 hours and receiving pre-pyloric enteral nutrition for more than 24 hours are included in this open-label cluster randomised parallel group non-inferiority trial. The participating centres are randomised allocated to continued enteral nutrition until extubation or 6-hour fasting (with concomitant gastric suctioning when feasible) prior to extubation. The primary outcome is extubation failure (ie, reintubation within 7 days following extubation). Ethics and dissemination This study has been approved by the national ethics review board (comite de protection, des personnes Sud Mediterranée III No 2017.10.02 bis) and patients are included after informed consent. Results will be submitted for publication in peer-reviewed journals. Trial registration number ClinicalTrials.gov Registry (NCT03335345).

\section{INTRODUCTION}

Extubation is a critical phase in the intensive care unit (ICU), with a risk of failure, that is, reintubation within the week following extubation, reported in about $15 \%$ of extubated patients. ${ }^{1}$ Extubation failure is strongly associated with the occurrence of nosocomial pneumonia. ${ }^{2}$ An important mechanism potentially implicated in this relationship is the aspiration of digestive secretions. ${ }^{3}$ The risk is particularly high after extubation, due to oropharyngeal dysfunction, lasting several hours after the removal of the endotracheal

\section{Strengths and limitations of this study}

This is the first pragmatic randomised multicentre study comparing fasting or continuous enteral feeding before extubation, a very common practice in intensive care units.

- We chose a strong patient-centred primary outcome: extubation failure.

- Cluster randomised design is well adapted to address this research question but required to design a pragmatic trial in order to improve feasibility of recruiting a large number of patients.

- This is an open study without blinding.

- The centres participating in this research are not all universities and therefore do not have the same impedance to research.

tube protecting the airways. Furthermore, in case of reintubation, general anaesthesia induction, which abolishes the protective reflexes of the airways, also represents a situation potentially at high-risk of aspiration. Therefore, many clinicians recommend a fasting period before extubation. ${ }^{4}$ This practice, based on guidelines for planned intubation and extubation related to scheduled surgical procedures, has never been evaluated for extubation of critically ill patients. Of note, fasting, beyond the extra workload (interrupting feeding, switching to intravenous nutrition delivery, adapting glycaemic control) may have deleterious effects in some patients, by delaying extubation to ensure obtaining an empty stomach and decreasing energy intake. Indeed, up to one-third of the caloric deficit observed in critically ill patients has been attributed to airway management procedures. ${ }^{56}$ Although of short duration, this deficit, particularly if repeated due to 
iterative fasting periods for planned extubation attempts that do not actually take place, could have a significant impact in some patients. Insufficient caloric intake may favour nosocomial infections and muscle wasting, therefore delaying weaning of mechanical ventilation and impact post-intensive care outcome. ${ }^{7-11}$ The hypothesis underlying the current trial is that the pursuit of enteral nutrition until extubation is not associated with an increased risk of failure compared with fasting prior to extubation.

\section{METHODS AND ANALYSIS \\ Trial design}

This is an open-label cluster randomised parallel group non-inferiority trial comparing fasting versus continuous enteral nutrition before extubation. Our main hypothesis is that the incidence of extubation failure will be similar in both groups. A total of 1100 patients will be included. The trial will be carried out in 22 centres, each centre will apply the same allocated strategy to all patients. This study is pragmatic in nature and will be conducted as an open trial. Blinding, although theoretically feasible, would be extremely cumbersome and costly to implement in this pragmatic academic trial.

\section{Justification of the cluster-based randomised design}

The study design, with cluster randomisation, is appropriate given the constraints and objectives of the study:

1. This design is suitable for studies analysing care practices. This study focuses on practices combining several medical and nursing procedures (fasting before extubation, suctioning via the gastric tube, possible parenteral compensation of the enteral fasting) rather than evaluation of a medical device or the administration of a single drug. Cluster randomisation, by attributing a single strategy to each of the participating centres, simplifies the implementation of the strategy, favouring adherence to the protocol. Individual randomisation, by obliging the care teams to apply different protocols to different patients, would be counterproductive.

2. The cluster randomised design is particularly suitable for a non-inferiority study. Indeed, any cross-arm contamination is highly deleterious because it may wrongly favour a conclusion of non-inferiority. The present study design, with parallel cluster randomisation, prevents interarm contamination, in that each unit will use only one of the strategies evaluated.

\section{Eligibility criteria}

\section{Inclusion criteria}

All patients will be included consecutively if they fulfil the following criteria:

- Patient hospitalised in a participating ICU.

- Invasive mechanical ventilation for at least 48 hours at the time of extubation decision.

- Pre-pyloric enteral nutrition for at least 24 hours at the time of extubation decision.
- Age $\geq 18$ years.

Exclusion criteria

- Decision not to reintubate the patient.

- Patient under guardianship or trusteeship.

- Pregnancy or breast feeding.

- Lack of social security coverage.

- Patient with a tracheostomy.

- Patient fed by post-pyloric enteral nutrition (jejunal tube).

- Patient already included in the trial.

\section{Outcomes}

Primary outcome

Proportion of patients experiencing extubation failure within 7 days following extubation. Extubation failure is defined as any reintubation or attempt of reintubation. Patients dying or tracheostomised within 7 days of extubation will be considered as extubation failures.

\section{Secondary outcomes}

- Semiquantitative measurement of the nursing workload: Visual Analogue Scale evaluating nursing workload during the 6 hours preceding extubation, during extubation and during the 6 hours following extubation.

- Proportion of patients with hypoglycaemia $(<0.7 \mathrm{~g} / \mathrm{L})$ and hyperglycaemia $(>2 \mathrm{~g} / \mathrm{L})$ the day before extubation, on the day of extubation and during the day after extubation.

- Proportion of patients with one or more episodes of nosocomial pneumonia in the ICU within the 14 days following extubation. Nosocomial pneumonia diagnosis will be adjudicated at the end of the study.

- Proportion of patients undergoing unprogrammed oxygen therapy within 7 days following extubation (ie, for respiratory failure, not as implemented routinely in the post-extubation period).

- Proportion of patients undergoing unprogrammed non-invasive ventilation within 7 days following extubation (ie, for respiratory failure, not as a systematic prophylactic measure in the post-extubation period).

- Proportion of patients with at least one episode of tachypnoea exceeding 30 cycles/min within 7 days following extubation.

- Proportion of patients with extubation failure within 7 days of extubation attributable to the aspiration of digestive secretions as determined by the investigator.

- Proportion of patients with extubation failure at 48 hours and 72 hours after extubation (same definition as the primary outcome).

- Total caloric intake (enteral and parenteral): the day before extubation, the day of extubation and the day after extubation.

- Duration of the episode of invasive mechanical ventilation before the extubation motivating study inclusion. For patients with several invasive mechanical ventilation episodes, this duration starts with the last 
intubation before extubation within the study. For patients intubated outside of the study unit, this duration starts at admission in the ICU participating in the study.

- Duration between the first successful spontaneous breathing trial and extubation.

- Duration of ICU stay.

- Proportion of patients dying in the ICU.

\section{Screening}

All ventilated patients referred to a participating clinical trial site will be considered for participation (screened). Inclusion and exclusion of patients will be reported according to the Consolidated Standards of Reporting Trials (CONSORT) statement.

\section{Intervention}

- Maximal gastric vacuity strategy: interruption of enteral nutrition at least 6 hours before extubation and concomitant continuous suctioning via the gastric probe (if of an appropriate size) for 6 hours before extubation. Choice to maintain the gastric probe after extubation is left to the discretion of the attending clinician. Choice and modalities used to reinitiate solid and/or liquid oral nutrition is left to the discretion of the attending clinician as well as parenteral caloric intake.

- Maintenance of caloric intake strategy: maintenance of the enteral caloric supply at the same flow rate until extubation. No suctioning via the gastric probe before, during or after extubation. Choice to maintain the gastric probe after extubation is left to the discretion of the attending clinician. Choice and modalities used to reinitiate solid and/or liquid oral nutrition is left to the discretion of the attending clinician as well as parenteral caloric intake.

\section{Statistics}

General

The study data will be analysed by CIC INSERM 1415, CHRU Tours, France, with R software, ${ }^{12}$ in accordance with the present pre-established statistical analysis plan. No intermediate analysis will be performed. A report on the statistical analysis will be written. We will comply with the recommendations of the CONSORT statement, and the three extensions relating to this trial (cluster randomised trials, non-pharmacological interventions and non-inferiority trials).

\section{Definition of the populations for analysis}

As this is a non-inferiority study, analyses will be performed according to both the intention-to-treat and per-protocol principles.

- Intention-to-treat analysis: all patients included in the trial, except those with consent withdrawal, will be taken into account in the analysis, regardless of adherence to or non-respect of the intervention.
- Per-protocol analysis: the population for the perprotocol analysis will be defined during a review of the data performed blind to the randomisation group.

In the maintenance of caloric intake group, patients will be included in the per-protocol analysis if the enteral nutrition flow rate has been maintained until extubation without a decrease in flow rate higher than $50 \%$ within 6 hours before extubation and enteral nutrition has not been completely interrupted more than $30 \mathrm{~min}$ within 6 hours before extubation.

In the maximal gastric vacuity group, patients will be included in the per-protocol analysis if no enteral nutrition has been administered during the 6 hours before extubation and continuous suctioning was performed continuously with no interruption of more than $30 \mathrm{~min}$ (among patients with a gastric probe of sufficient diameter for suctioning). Patients with unintentional extubation before 6 hours of continuous fasting and gastric probe suctioning will be included in the per-protocol analysis as this may represent a side effect of the strategy and not a protocol violation.

\section{Description of patient characteristics at inclusion}

The groups resulting from randomisation will be described with the following statistics (no statistical tests will be performed): for qualitative variables, absolute numbers and percentages, and, for quantitative variables, mean and SD or median and IQR, depending on the data distribution. The characteristics of the patients at inclusion will be reported at the patient level or at the cluster level when appropriate.

The variables identified will be age, sex, body mass index, SAPS II, type of admission (unscheduled surgical, scheduled surgical, medical), the comorbid condition, the number of spontaneous breathing trial failure, administration of steroids before extubation, the cough evaluation, the tracheal secretion evaluation and the risk factors of extubation failure (stroke, neuromuscular disease, central nervous system infection, severe head trauma, diaphragmatic dysfunction, depressive disorder, delirium).

\section{Analysis of the primary endpoint}

In this non-inferiority trial, the principal analysis will estimate a CI for the difference between groups and will compare one of the limits of this CI with the zone of noninferiority, fixed a priori at $10 \%$. The extubation failure rates will be estimated in each group, the difference in extubation failure rates between the two groups will be estimated, and a two-tailed 95\% CI with corrected variances to take into account the cluster design will be calculated. ${ }^{13}$ The upper limit of this CI will then be compared with 0.10 . The relative risk will also be presented using a mixed-effect log-binomial model. In the particular case where the $95 \%$ CI for the difference lies entirely above or below zero, then there is evidence of superiority and we will calculate the $p$ value associated with a test of superiority at the $5 \%$ level $(\mathrm{p}<0.05)$. 
The principal analysis will be completed by an estimation of the intraclass correlation coefficient. This coefficient will be estimated independently in each of the two groups, with the Fleiss-Cuzick estimator. ${ }^{14}$

\section{Analysis of the secondary endpoints}

All estimators will be accompanied by a 95\% CI taking into account clustering.

- Incidence of nosocomial pneumonia in intensive care during the 14 days following the first extubation: this endpoint will be the object of adjudication by an independent centralised committee blind to the randomisation group. This endpoint is an integer count and will therefore be analysed with a mixed-effect Poisson model, taking the duration of observation as an offset and considering a random effect of cluster.

- Total caloric supply (enteral and parenteral) the day before extubation, the day of extubation and the day after extubation: this quantitative endpoint will be analysed with a linear mixed-effects model, with a random effect for each cluster.

- Nursing workload: this quantitative endpoint will be analysed with a linear mixed-effects model, with a random effect for each cluster.

- Extubation failure attributable to the inhalation of gastric fluids: this binary endpoint will be analysed according to the same principles as the principal endpoint.

- Extubation failure at 48 and 72 hours after extubation: this binary endpoint will be evaluated according to the same principles as the principal endpoint.

- Duration of invasive mechanical ventilation between intubation in intensive care (or admission to intensive care for patients intubated before admission) and the first extubation within the study: this time-to-event endpoint will be analysed with a mixed-effects Cox regression, with a random effect for each cluster. ${ }^{15}$

- Time between the first successful spontaneous breathing trial and extubation: this time-to-event endpoint will be analysed with a mixed-effects Cox regression, with a random effect for each cluster. ${ }^{15}$

- Incidence of hyperglycaemia and hypoglycaemia the day before extubation, the day of extubation and the day after extubation: these binary endpoints will be analysed according to the same principles as the principal endpoint.

- Duration of stay in intensive care and in the hospital: the duration will be analysed with a competing risks model, with death as an event in competition with alive discharge from the ICU or hospital. The model will be a population average regression which takes into account the correlation across individuals within a cluster. ${ }^{16}$

- Mortality in intensive care: this binary endpoint will be evaluated according to the same principles as the principal endpoint.

- Respiratory events after extubation, unplanned use of oxygen therapy, unplanned non-invasive ventilation and occurrence of tachypnoea: these events will be analysed by the same approach as for nosocomial pneumonia.

\section{Sample size}

This study will require 1100 patients.

Based on the hypothesis of an incidence of extubation failure of $16 \%$ in the control group, with a non-inferiority margin of $10 \%$, a type I error of $2.5 \%$ and a power of $80 \%$, we would need to include 210 patients per group in an individual randomisation framework. ${ }^{17}$ The cluster randomisation makes it necessary to apply an inflation coefficient taking into account the correlation between patients from the same cluster. For this study, we will assume an intraclass correlation coefficient of $0.03{ }^{18}$ The principal endpoint is an 'outcome' (ie, it concerns the response of the patient to the management applied) rather than a 'process' (ie, dealing with the practices of the personnel in the cluster). For endpoints of this type, Campbell and coworkers reported a median intraclass correlation coefficient of $0.03{ }^{18}$ We will also take into account differences in the sizes of the centres, using the approach of Eldridge and coworkers based on the coefficient of variation for cluster size. ${ }^{16}$ For this, we will make use of the number of beds per centre, which is probably a good proxy for the number of patients eligible per centre. The coefficient of variation for the number of beds is 0.31 . Finally, the planned number of clusters for this trial is 22. The mean number of patients per cluster can thus be obtained with the following equation: $11 \times \mathrm{p}=210 \times\left[1+\left(\left(0.31^{2}+1\right) \mathrm{p}-1\right) \times 0.03\right]$.

Which gives $\mathrm{p}=49.8$.

We will therefore need to include a mean of 50 patients per centre, resulting in a total of 1100 patients being required.

\section{Randomisation}

According to cluster randomisation, the units will be randomly assigned, before the start of the study, to the study arm to which they will supply included patients. Each unit will apply the strategy assigned (maximal gastric vacuity or maintenance of caloric intake strategy) to all the patients included in the study within the unit. Randomisation will be stratified according to whether the centres are university hospitals or not and according to whether most of the patients in the unit come from medico-surgical or surgical departments.

\section{Course of the study}

The duration of participation for each patient will be the duration of their stay in intensive care, up to a maximum of 28 days.

\section{Extubation visit}

This visit will take place at the time of extubation and gather data linked to extubation, data of the preceding day and the period immediately after extubation.

Collected information: caloric supply over 24-hour periods, time from intubation to extubation and time 
from the first successful spontaneous breathing trial to extubation, incidence of hypoglycaemia and hyperglycaemia, maintenance or removal of the gastric probe immediately after extubation, and time to oral refeeding after extubation. The following risk factors for extubation failure will be recorded: neuromuscular disease, acute cerebral vascular disease (ischaemic or haemorrhagic), neuropathy, myopathy, infection of the central nervous system, serious head trauma, diaphragm dysfunction, cognitive dysfunctions, anxiety-depression syndrome, delirium and Richmond Agitation Sedation Scale score in the 24 hours after extubation. ${ }^{19}$

\section{Visit on the day following extubation}

Collected information: caloric supply over the last 24 hours, incidence of hypoglycaemia and hyperglycaemia, time to oral refeeding if not already initiated and occurrence of reintubation. Subsequent visits will take place only for patients in intensive care on the day concerned. The decision to discharge the patient from intensive care will be left to the discretion of the attending clinician.

\section{Daily visits during the 7 days following extubation}

Collected information: any occurrence of reintubation, nosocomial pneumonia or respiratory events (maximal oxygen rate, non-invasive ventilation, maximal respiratory frequency).

The cause of any reintubation will be noted (acute pulmonary oedema, obstruction/oedema of the airways, inhalation of fluids, new pneumonia, atelectasis, pleurisy/pleural effusion, pneumothorax, bronchial congestion, ineffective cough, respiratory muscle weakness, alveolar hypoventilation, hypercapnic coma, septic shock, cardiogenic shock, central neurological causes/encephalopathy, surgical procedure, other).

\section{Daily visits beyond the 7th day, up to the 14th day after extubation}

Any occurrence of nosocomial pneumonia will be recorded over this period.

\section{Duration of the study}

Patients are expected to be included during an 18-month period starting in April 2018.

- 2017: protocol approval from the ethics committee and trial tools development (case report form, randomisation system).

- 2018-2019: inclusion of patients.

- 2020: cleaning and closure of the database. Data analysis.

- 2021: writing of the manuscript and submission for publication in a peer-reviewed journal.

The duration of participation for each patient in the protocol corresponds to their stay in intensive care, up to a maximum of 28 days. The first patient included dates from April 2018.

\section{Feasibility of the study}

Extubation is a routine clinical practice performed among all the ICU patients treated with invasive mechanical ventilation after recovery of critical illness. In most intensive care departments, more than $50 \%$ of the patients undergo intubation and thus potentially extubation. About half of these patients are ventilated for more than 48 hours and most receive enteral nutrition which renders them eligible to the study. Thus, a department admitting 50 patients per month is likely to include about 10 patients per month. The hypothesis of two inclusions per month and per centre is therefore conservative. These hypotheses concerning recruitment were confirmed in a feasibility study performed in 10 representative centres scheduled to take part in this project. This feasibility study was performed over a short period (15 days) in the summer of 2016, and was not, therefore, representative of winter activity, which is generally greater in intensive care departments. Nevertheless, this study demonstrated the validity and pertinence of the inclusion criteria for this study. Indeed, over this period, 48 patients satisfied the inclusion criteria among the 10 centres. By extrapolating this figure to 22 centres, we can foresee a monthly recruitment rate of 200 patients. The primary endpoint is simple to record: extubation failure by definition leads to a reintubation attempt. Reintubation is a complex procedure necessitating the administration of specific drugs, systematically noted in the patient's medical records. It is, therefore, a very simple criterion to record, particularly as it is assessed only during the 7 days following extubation.

This project will be performed in the framework of a collaboration between the ICUs of the West of France and the corresponding university hospitals (college interrégional Ouest de Médecine Intensive Réanimation).

Finally, the study has received the support of two international intensive care clinical research networks: the Clinical Research in Intensive Care and Sepsis-Trial Group for Global Evaluation and Research in Sepsis ( www.triggersep.org) and the Réseau Européen de Recherche en Ventilation artificielle (the European Artificial Ventilation Research Network: http://www.reseau-reva.org).

\section{Data collection and management \\ Data collection}

All the information required for the protocol will be collected in the electronic case report form (eCRF), which can be accessed solely via a secured internet connection. The investigators will be provided with a document to help them use this tool.

\section{Data management}

Data management in this study will be the responsibility of a data manager from CIC INSERM 1415, CHRU Tours, France. The eCRF will be developed with Ennov Clinical software. Data will be managed according to the standard operating procedures in force at CIC INSERM 1415. The data will be input at the investigating centres and queries will be formulated by the data manager according to a 
consistency control plan established during the design of the eCRF.

A blind review of the data will be performed before the database is locked. The database will be locked according to standard operating procedures in force at CIC INSERM 1415 , and the data will be extracted for statistical analyses.

\section{Patient and public involvement}

No patient involved. Indeed, it seems difficult to us to involve patients in the drafting of the protocol on a practice evaluation, the debate of which greatly divides the medical community.

This study respects the Standard Protocol Items: Recommendations for Interventional Trials recommendations and checklist. ${ }^{20} 21$

\section{ETHICS AND DISSEMINATION}

This study has been approved by the national ethics review board (Comité de protection des personnes Sud Mediterranée III No 2017.10.02 bis) and complies with the current revision of the Declaration of Helsinki, the International Conference on Harmonisation Note for Guidance on Good Clinical Practice and the applicable French regulatory requirements. The patients, or their proxy, will be informed of their right to refuse participation in the study. In all cases, before inclusion in the study, express informed consent will be obtained from the patient, or, if this is not possible, from a proxy (legal representative or next of kin), and the process documented in the patient's medical file, according to French law. In all cases, the patient will be informed, and consent obtained as soon as possible. Subjects can oppose their participation secondarily and request to be withdrawn from the study at any time and for any reason. The investigator can stop the study intervention temporarily or definitively for any reason in the best interests of the subject.

Research assistants will regularly monitor all the centres on site to check adherence to the protocol and the accuracy and completeness of the data recorded.

The results will be submitted for publication in peerreviewed journals.

\section{DISCUSSION}

The expected results of this study should have an immediate impact on the management of a very large number of patients in ICUs around the world in that they will provide a concrete response and a high level of evidence concerning a daily routine clinical practice. If the study hypothesis proves correct, it will no longer be necessary to impose a fasting period to patients before extubation, and this will immediately simplify patient management in units with such a practice. A series of secondary benefits may accrue to patients: shorter time to extubation, decreased nursing workload and increased caloric intake.

Conversely, if the study hypothesis proves incorrect, and the continuation of enteral nutrition before extubation is inferior to the maximal gastric vacuity strategy, this study will also have an immediate clinical impact in ICUs not yet imposing a fasting period before extubation to patients. It will also open up new fields of investigation into the optimal mode of fasting before extubation.

The cluster randomised design of the study should make it possible to transfer the results rapidly into clinical practice at a large number of ICUs.

To the best of our knowledge, fasting and suctioning of the gastric contents via a gastric tube has never been rigorously evaluated in the context of extubation in adult critical care.

A declarative study among 80 critical care centres in North America (postal survey) showed that the fasting procedures before extubation were very heterogeneous between centres with duration of fasting varying from the absence of fasting, to up to 8 hours of fasting before extubation. ${ }^{22}$

The Ambroisie Project is an investigator-initiated pragmatic randomised controlled trial undertaken to test the hypothesis that pursuing enteral nutrition until extubation is non-inferior to a strategy of maximal gastric vacuity before extubation in the ICU. If the hypothesis proves correct, continued enteral nutrition until extubation could be recommended and would simplify the management of patients.

\section{Author affiliations}

${ }^{1}$ Polyvalent Resuscitation, Le Mans Hospital Center, Le Mans, France

${ }^{2}$ Medical Intensive Care Unit, Orleans Regional Hospital Center La Source Hospital, Orleans, France

${ }^{3}$ Intensive Care Unit, Cholet Hospital Center, Cholet, France

${ }^{4}$ Cardiac Resuscitation Service, Pitié Salpêtrière University Hospital, Paris, France

${ }^{5}$ INSERM CIC 1415, Tours, France

${ }^{6}$ Multipurpose Resuscitation, Le Mans Hospital Center, Le Mans, France

${ }^{7}$ INSERM 1246-SPHERE, University of Tours, Tours, France

${ }^{8}$ Intensive Resuscitation Medicine, CHU Nantes, Nantes, France

${ }^{9}$ Médecine Intensive Réanimation, CHU de Poitiers, 2 rue la Milétrie, 86021, Poitiers, Poitiers, France

${ }^{10} \mathrm{CIC} 1402$ ALIVE, INSERM, Poitiers, France

${ }^{11}$ Université de Tours, Université de Nantes, INSERM, SPHERE U1246, Tours, France; INSERM CIC1415, CHRU de Tours, 2 boulevard Tonnellé, Tours cedex 9, France

${ }^{12}$ Intensive Resuscitation Medicine, CHRU Tours, Tours, France

${ }^{13} \mathrm{Center}$ for the Study of Respiratory Pathologies, U1100, INSERM, Tours, France

Contributors ML, M-AN, JA, NH, AWT, BG, JR, ET and SE designed the study. $M L, M-A N, J A, E T$ and SE wrote the study protocol. ARebion, ARobert, BG and ET planned the data analysis and management. All authors reviewed the manuscript for important intellectual content and approved the final version submitted.

Funding This work was supported by a grant of the French Ministry of Health (Projet hospitalier de recherche clinique, appel d'offre inter-régional Ouest №. API16/A/063).

Competing interests SE declares receiving consulting fees, unrestricted research grants and equipment research support from Aerogen, unrestricted research grant from Fisher \& Paykel, unrestricted research grant from Hamilton medical, and consulting fees from La Diffusion Technique Française.

Patient and public involvement Patients and/or the public were not involved in the design, or conduct, or reporting, or dissemination plans of this research.

Patient consent for publication Not required.

Provenance and peer review Not commissioned; externally peer reviewed.

Open access This is an open access article distributed in accordance with the Creative Commons Attribution Non Commercial (CC BY-NC 4.0) license, which 
permits others to distribute, remix, adapt, build upon this work non-commercially, and license their derivative works on different terms, provided the original work is properly cited, appropriate credit is given, any changes made indicated, and the use is non-commercial. See: http://creativecommons.org/licenses/by-nc/4.0/.

\section{ORCID iDs}

Mickael Landais http://orcid.org/0000-0001-5320-4013

Mai-Anh Nay http://orcid.org/0000-0002-6116-4987

Jean Reignier http://orcid.org/0000-0002-3768-3496

Elsa Tavernier http://orcid.org/0000-0003-0798-1182

\section{REFERENCES}

1 Thille AW, Harrois A, Schortgen F, et al. Outcomes of extubation failure in medical intensive care unit patients. Crit Care Med 2011;39:2612-8.

2 Maggiore SM, Battilana M, Serano L, et al. Ventilatory support after extubation in critically ill patients. Lancet Respir Med 2018;6:948-62.

3 , American Thoracic Society, Infectious Diseases Society of America. Guidelines for the management of adults with hospital-acquired, ventilator-associated, and healthcare-associated pneumonia. Am J Respir Crit Care Med 2005;171:388-416.

4 Smith I, Kranke P, Murat I, et al. Perioperative fasting in adults and children: guidelines from the European Society of Anaesthesiology. Eur J Anaesthesiol 2011;28:556-69.

5 O'Meara D, Mireles-Cabodevila E, Frame F, et al. Evaluation of delivery of enteral nutrition in critically ill patients receiving mechanical ventilation. Am J Crit Care 2008;17:53-61.

6 De Jonghe B, Appere-De-Vechi C, Fournier M, et al. A prospective survey of nutritional support practices in intensive care unit patients: what is prescribed? What is delivered? Crit Care Med 2001;29:8-12.

7 Faisy C, Lerolle N, Dachraoui F, et al. Impact of energy deficit calculated by a predictive method on outcome in medical patients requiring prolonged acute mechanical ventilation. Br J Nutr 2009;101:1079-87.

8 Villet S, Chiolero RL, Bollmann MD, et al. Negative impact of hypocaloric feeding and energy balance on clinical outcome in ICU patients. Clin Nutr 2005;24:502-9.
9 Rubinson L, Diette GB, Song X, et al. Low caloric intake is associated with nosocomial bloodstream infections in patients in the medical intensive care unit. Crit Care Med 2004;32:350-7.

10 Dvir D, Cohen J, Singer P. Computerized energy balance and complications in critically ill patients: an observational study. Clin Nutr 2006;25:37-44.

11 Alberda C, Gramlich L, Jones N, et al. The relationship between nutritional intake and clinical outcomes in critically ill patients: results of an international multicenter observational study. Intensive Care Med 2009;35:1728-37.

12 R Core Team. R: a language and environment for statistical computing. Vienna, Austria: R Foundation for Statistical Computing, 2014. http://www.R-project.org/

13 Donner A, Shoukri MM, Klar N, et al. Testing the equality of two dependent kappa statistics. Stat Med 2000;19:373-87.

14 Zou G, Donner A. Confidence interval estimation of the intraclass correlation coefficient for binary outcome data. Biometrics 2004;60:807-11.

15 Therneau T, Grambsch P. Modeling survival data: extending the Cox model. New York: Springer-Verlag, 2000.

16 Zhou B, Fine J, Latouche A, et al. Competing risks regression for clustered data. Biostatistics 2012;13:371-83.

17 Thille AW, Richard J-CM, Brochard L. The decision to extubate in the intensive care unit. Am J Respir Crit Care Med 2013;187:1294-302.

18 Campbell MK, Fayers PM, Grimshaw JM. Determinants of the intracluster correlation coefficient in cluster randomized trials: the case of implementation research. Clin Trials 2005;2:99-107.

19 Sessler CN, Gosnell MS, Grap MJ, et al. The Richmond AgitationSedation scale: validity and reliability in adult intensive care unit patients. Am J Respir Crit Care Med 2002;166:1338-44.

20 Chan A-W, Tetzlaff JM, Altman DG, et al. Spirit 2013 statement: defining standard protocol items for clinical trials. Ann Intern Med 2013;158:200-7.

21 Chan A-W, Tetzlaff JM, Gøtzsche PC, et al. Spirit 2013 explanation and elaboration: guidance for protocols of clinical trials. BMJ 2013;346:e7586.

22 Schneider JA, Lee YJ, Grubb WR, et al. Institutional practices of withholding enteral feeding from intubated patients. Crit Care Med 2009;37:2299-302. 